\title{
EFEITO DE ADIÇÃO DE DIFERENTES SAIS DE CÁLCIO NAS CARACTERÍSTICAS DA MASSA E NA ELABORAÇÃo DE PÃO FRANCÊS' ${ }^{1}$
}

\author{
Shizuko KAJISHIMA², Matilde PUMAR ${ }^{3}$, Rogério GERMANI ${ }^{4, *}$
}

\begin{abstract}
RESUMO
O objetivo deste trabalho foi estudar os efeitos da adição do sulfato de cálcio, carbonato de cálcio e fosfato de cálcio dibásico nas características físicas e reológicas da farinha de trigo e na qualidade de pão francês. Utilizou-se a quantidade de sais de cálcio que correspondesse a $100 \%$ da IDR de adulto por $100 \mathrm{~g}$ de pão. Na farinha foram realizadas análises de cor, teor de glúten, farinografia, alveografia e extensigrafia. O pão francês elaborado foi analisado quanto a seu volume específico e conceito global. O enriquecimento com cálcio melhorou a cor da farinha e os resultados satisfatórios obtidos na reologia das massas e com os pães mostraram que se pode utilizar qualquer uma das três fontes para se elaborar pão enriquecido com cálcio.

Palavras-chave: pão francês; enriquecimento de farinha; sulfato de cálcio; carbonato de cálcio; fosfato de cálcio.
\end{abstract}

\section{SUMMARY}

EFFECT OF ADDITION OF DIFERENT SOURCES OF CALCIUM ON THE FRENCH BREAD MAKING. The objetive of this work was to study the effect of addition of calcium sulfate, calcium carbonate and calcium phosphate on the dough flour physical and rheological characteristics and on the french bread quality. The calcium salts were used in the level of $100 \%$ of adult RDA for $100 \mathrm{~g}$ of bread. Color, gluten content, farinograph, alveograph and extensigraph tests were carried out on the flour. On the bread, specific volume and global score were determined. The color of the flour was improved with calcium addition. The rheological properties and the bread volume and global score were not significantly influenced by the addition of calcium at $100 \%$ of RDA.

Keywords: french bread; flour enrichment; calcium sulfate; calcium carbonate; calcium phosphate.

\section{1 - INTRODUÇÃO}

A indústria alimentícia tem particular interesse na melhoria do valor nutricional dos produtos industrializados devido as perdas dos teores de vitaminas e minerais durante o beneficiamento desses alimentos [4].

Entre os alimentos, os cereais são os mais consumidos mundialmente, sendo o trigo, o que tem maior aceitação [5]. Segundo a Associação Brasileira de Indústria de Panificação - ABIP [2], o pão é um dos alimentos mais difundidos e se constitui em uma das principais fontes calóricas da dieta do brasileiro. Segundo IBGE [12], o consumo anual per capita de pão francês é de $18,4 \mathrm{~kg}$, perdendo somente para o arroz polido, que é de $26,5 \mathrm{~kg}$.

VANNUCCHI et al. [18] consideraram o baixo consumo de cálcio como um problema nutricional presente e potencial no país, causando problemas ósseos como osteosporose no adulto e raquitismo nas crianças.

Como o pão francês tem amplo consumo pela população brasileira, o mesmo tem grande potencial de servir como veículo para promover uma maior ingestão de cálcio.

A quantidade de sais de cálcio empregada no enriquecimento pode ser um problema, pois são necessárias

1. Recebido para publicação em 16/10/2001. Aceito para publicação em 09/09/2002 (000763).

2. Departamento de Tecnologia Rural, Centro de Formação de Tecnólogos,

Universidade Federal da Paraíba, Bananeiras - PB, CEP 58. 220-000.

2.Fac.de Nutrição/UFF. shinje@uol.com.br-R. São Paulo 30/4andar,

Niterói, RJ, 24.015-110

3. Instituto de Nutrição da UERJ.

4. EMBRAPA - Agroindústria de Alimentos, Rio de Janeiro, RJ.

* A quem a correspondência deve ser enviada. de 2,2g a 3,5g de sais para se alcançar a IDR (Ingestão Diária Recomendada) deste mineral. Essas grandes quantidades podem alterar as características da massa e do pão produzido.

As formas de cálcio mais indicadas para utilização em enriquecimento são os sais orgânicos de cálcio, devido a sua solubilidade, e por conseqüência, melhor absorção pelo organismo. Dentre os sais de cálcio utilizados para enriquecimento da farinha de trigo e produtos de cereais temos: carbonato de cálcio, fosfato de cálcio e sulfato de cálcio [7]. O carbonato de cálcio é amplamente aplicado devido a concentração deste mineral ser de $40 \%$ e, economicamente mais barato [15]. O sulfato de cálcio, com 29\% de concentração, apresentou melhor biodisponibilidade, em estudo experimental realizado em animais [16].

A National Academy of Sciences/National Research Council - NAS/NRC [13], em relação a fortificação de cálcio em grãos e produtos de cereais, recomendou $198,2 \mathrm{mg} / 100 \mathrm{~g}$ do grão ou produto, que corresponde a $5,6 \%$ do IDR deste mineral. O padrão americano (Federal Enrichment Standard) sugeriu a adição de cálcio aos niveis de $132,3 \mathrm{mg} / 100 \mathrm{~g}$ de pão e $211,6 \mathrm{mg} / 100 \mathrm{~g}$ de farinha [14]. Já RANUM [17] recomendou $105 \mathrm{mg} / 100 \mathrm{~g}$ de farinha, ou seja, um menor nivel, justificando que o pão em geral já contém na sua formulação propionato de cálcio, lactato de cálcio ou muitas vezes, cálcio oriundo da água. Portanto, dependendo da formulação do pão, este já pode conter de 10 a $75 \%$ do cálcio recomendado para o seu enriquecimento.

Segundo GERMANI et al. [10], a presença de sais de cálcio podem ter efeito sobre o fortalecimento do glúten e atuam, ligeiramente, como nutriente para o fermento. 
Estudos em pão de forma, mostraram que quantidades em torno de 300mg de cálcio em 453,6g (1 libra) de pão não provocam efeito desfavorável na qualidade do produto [16].

Desta forma, este trabalho teve como objetivo avaliar os efeitos de diferentes sais de cálcio nas características da massa e pão francês obtido de farinhas enriquecidas com esse nutriente.

\section{2 - MATERIAL E MÉTODOS}

Foi utilizada farinha de trigo comercial de um mesmo lote obtida em moinho na cidade do Rio de Janeiro e as fontes de cálcio utilizadas para enriquecimento foram: o sulfato de cálcio, $\mathrm{CaSO}_{4} \cdot 2 \mathrm{H}_{2} \mathrm{O}(232 \mathrm{mgCa} / \mathrm{g})$; carbonato de cálcio, $\mathrm{CaCO}_{3}(400 \mathrm{mgCa} / \mathrm{g})$ e, fosfato de cálcio dibásico, $\mathrm{CaHPO}_{4}(294 \mathrm{mgCa} / \mathrm{g})$ fornecidos pela VETEC Química Fina Ltda. Estes produtos são permitidos desde 1981 como aditivos alimentares [6].

Com quantidade de cálcio suficiente para se obter $100 \%$ da IDR [3] deste elemento (800mg) por $100 \mathrm{~g}$ de pão francês, avaliou tanto a qualidade tecnológica da farinha como do pão produzido. Isto corresponde a 3,44g, 2,0Og e 2,72g de $\mathrm{CaSO}_{4} 2 \mathrm{H}_{2} \mathrm{O}, \mathrm{CaCO}_{3}$ e $\mathrm{CaHPO}_{4}$, respectivamente, que foram adicionados a cada $74 \mathrm{~g}$ de farinha (presente em 100g de pão).

Como parâmetro de qualidade tecnológica da farinha foram analisados a cor, através do colorimetro KENTJONES \& MARTINS (Flour color grade series 2), os teores de glúten (úmido e seco) e o índice de glúten segundo o método 38-12 da AACC [1], utilizando o aparelho GLUTOMATIC.

As características de mistura foram determinadas segundo o método 54-21 da AACC [1], utilizando o farinógrafo Brabender, e as características de extensão determinadas através de teste de alveografia, segundo o método 54-30A da AACC [1] e pelo extensígrafo Brabender, segundo método 54-10 da AACC [1], utilizando, neste último, somente o tempo de descanso de 135 minutos.

O processo de panificação foi baseado no descrito por EL-DASH, MAZZARI \& GERMANI [9], utilizando masseira lenta. A formulação de pão francês utilizada nos experimentos foi estipulada após ensaios prévios (Tabela 1). Para avaliação do pão foi utilizada média ponderal, onde foram atribuídos peso 60 para o volume específico e 10 para cada um dos atributos: cor da crosta, simetria, cor do miolo, grão e textura, totalizando ao final um máximo de 100 pontos. Este escore foi então convertido em conceito global determinado como: excelente (91-100), muito bom (81-90), bom (71-80), regular $(61-70)$ e deficiente $(<60)$ segundo GERMANI \& BENASSI [11]. Com exceção do peso e do volume do pão, as outras avaliações foram feitas por um especialista no assunto.

O volume e peso dos pães foram medidos logo após o resfriamento, conforme recomendado por EL-DASH; CAMARGO \& DIAZ [8].

Todos os ensaios foram conduzidos em triplicata e os dados foram submetidos a análise de variância (ANOVA), com o procedimento GLM (General Linear Model) do pacote estatístico SAS (Statistical Analysis System). Para comparação entre médias, foi utilizado o teste de Tukey ao nivel de significância de $5 \%$ de probabilidade.

TABELA 1. Formulação do pão francês.

\begin{tabular}{lc}
\hline & QUANTIDADE \\
\hline INGREDIENTES & $3000 \mathrm{~g}$ \\
Sal & $60 \mathrm{~g}$ \\
Fermento biológico fresco & $36 \mathrm{~g}$ \\
Gordura hidrogenada & $30 \mathrm{~g}$ \\
Ácido ascórbico & $150 \mathrm{ppm}$ \\
Condicionador de massa & $30 \mathrm{~g}$ \\
Sulfato, carbonato ou fosfato de cálcio & 140,$4 ; 81,9 \mathrm{ou} 111,3 \mathrm{~g}$ \\
Água & $1750 \mathrm{~mL}$ \\
\hline
\end{tabular}

\section{3 - RESULTADOS E DISCUSSÃo}

Entre os sais utilizados, observou-se que a homogeneização do $\mathrm{CaHPO}_{4}$ na farinha foi a mais fácil. Já para o sulfato de cálcio, foi necessário que este fosse peneirado em malha no 45 (abertura de 0,35 mm) para uniformizar o tamanho do sal e desagregar os grumos antes da homogeneização com a farinha. Com o carbonato de cálcio o aparecimento dos grumos se deu durante a homogeneização com a farinha. Neste caso foi necessário passar esta farinha enriquecida por um peneiramento em malha $\mathrm{n}^{\circ} \mathrm{45}$, e então novamente repetida a operação de homogeneização. Pela análise da cor houve diferença significativa entre a farinha Padrão e as enriquecidas. O valor da cor diminuiu com a adição de sais de cálcio (quanto menor o valor mais clara a cor) sendo que aquela com $\mathrm{CaCO}_{3}$ resultou na farinha mais branca, como pode ser observado na Tabela 2.

TABELA 2. Cor da farinha de trigo Padrão e das enriquecidas de diferentes sais de cálcio.

\begin{tabular}{|c|c|}
\hline INGREDIENTES & QUANTIDADE \\
\hline Farinha padrão & $3000 \mathrm{~g}$ \\
\hline Sal & $60 \mathrm{~g}$ \\
\hline Fermento biológico fresco & $36 \mathrm{~g}$ \\
\hline Gordura hidrogenada & $30 \mathrm{~g}$ \\
\hline Ácido ascórbico & 150ppm \\
\hline Condicionador de massa & $30 \mathrm{~g}$ \\
\hline Sulfato, carbonato ou fosfato de cálcio & 140,$4 ; 81,9$ ou $111,3 \mathrm{~g}$ \\
\hline Áqua & $1750 \mathrm{~mL}$ \\
\hline
\end{tabular}

Houve diferença significativa no teor de glúten úmido entre as quatro farinhas, embora essas não tenham sido relevantes. Não houve diferença entre a farinha Padrão e a adicionada de fosfato de cálcio. Já as enriquecidas de sulfato e carbonato de cálcio, os valores foram levemente 
inferiores a da Padrão. Por outro lado, os valores de glúten seco e índice de glúten não se alteraram significativamente para as quatro farinhas (Tabela 3).

TABELA 3. Teores de glúten úmido, glúten seco e índice de glúten da farinha de trigo Padrão e das enriquecidas de diferentes sais de cálcio.

\begin{tabular}{lccc}
\hline AMOSTRAS & $\begin{array}{c}\text { GLÚTEN } \\
\text { ÚMIDO } \\
(\%)\end{array}$ & $\begin{array}{c}\text { GLÚTEN } \\
\text { SECO } \\
(\%)\end{array}$ & $\begin{array}{c}\text { INDICE DE } \\
\text { GLÚTEN } \\
(\%)\end{array}$ \\
\hline Farinha Padrão & $24,1 \mathrm{a}^{*}$ & $8,1 \mathrm{a}$ & $98 \mathrm{a}$ \\
Farinha com CaSO ${ }_{4} \mathrm{H}_{2} \mathrm{O}$ & $22,0 \mathrm{~b}$ & $8,2 \mathrm{a}$ & $98 \mathrm{a}$ \\
Farinha com $\mathrm{CaCO}_{3}$ & $22,6 \mathrm{ab}$ & $8,1 \mathrm{a}$ & $99 \mathrm{a}$ \\
Farinha com $\mathrm{CaHPO}_{4}$ & $24,1 \mathrm{a}$ & $8,9 \mathrm{a}$ & $99 \mathrm{a}$ \\
\hline
\end{tabular}

médias das colunas seguidas de letra igual não são significativamente diferentes $(\mathrm{p} \leq 5 \%)$, pelo teste de Tukey.

Pela avaliação farinográfica, a adição dos sais de cálcio, sulfato e fosfato, diminuíram significativamente a absorção de água da farinha, enquanto que a adição de carbonato de cálcio não causou alteração significativa. Por outro lado, a estabilidade foi significativamente aumentada quando se adicionou qualquer um dos sais de cálcio. Este aumento, segundo CIACCO \& CHANG [5] é devido a presença de íons, dando maior estabilidade as pontes de enxofre. O maior valor obtido foi com o fosfato seguido pelo carbonato e sulfato (Tabela 4). A adição dos sais de cálcio não alterou significativamente os tempo de desenvolvimento da massa, valores de indice de tolerância da mistura e valor do valorimetro da farinha de trigo em estudo.

TABELA 4. Farinografia ${ }^{1}$ da farinha de trigo Padrão e das enriquecidas de diferentes sais de cálcio.

\begin{tabular}{|c|c|c|c|c|c|}
\hline AMOSTRAS & $\begin{array}{c}\text { ABS } \\
(\%)\end{array}$ & $\begin{array}{r}\text { TDM } \\
\text { (min) }\end{array}$ & $\begin{array}{c}\text { EST } \\
\text { (min) }\end{array}$ & $\begin{array}{l}\text { ITM } \\
\text { (UF) }\end{array}$ & W \\
\hline Farinha Padrão & $58,7 a^{*}$ & $15,2 a$ & $14,0 \mathrm{~d}$ & $22,5 \mathrm{ba}$ & $89,5 a$ \\
\hline Farinha com CaSO ${ }_{4} 2 \mathrm{H}_{2} \mathrm{O}$ & $57,2 b$ & $15,2 a$ & $19,5 c$ & $32,5 a$ & $89,5 a$ \\
\hline Farinha $\operatorname{com} \mathrm{CaCO}_{3}$ & $58,8 a$ & $15,0 a$ & $22,6 b$ & 27,5 ba & $89,5 a$ \\
\hline Farinha com CaHPO 4 & $57,5 \mathrm{~b}$ & $16,0 \mathrm{a}$ & $41,2 \mathrm{a}$ & $20,0 \mathrm{~b}$ & $91,0 \mathrm{a}$ \\
\hline
\end{tabular}

Os resultados das avaliações de alveografia das farinhas Padrão e enriquecidas com os diversos sais de cálcio são mostrados na Tabela 5 .

A farinha enriquecida com o $\mathrm{CaCO}_{3}$ foi significativamente mais tenaz que as demais farinhas enriquecidas com os outros dois sais de cálcio, e estas não foram significativamente diferentes da farinha Padrão. Os demais parâmetros não foram significativamente afetados pela adição dos sais de cálcio.

Como mostrados na Tabela 6 , não houveram diferenças significativas em qualquer dos resultados obti- dos pela extensigrafia, ou seja, os sais de cálcio pouco afetaram as propriedades de extensão da massa, avaliadas neste equipamento.

TABELA 5. Alveografia ${ }^{1}$ da farinha de trigo Padrão e das enriquecidas de diferentes sais de cálcio.

\begin{tabular}{|c|c|c|c|c|}
\hline AMOSTRAS & $\begin{array}{c}\mathbf{P} \\
\left(\mathrm{mmH}_{2} \mathrm{O}\right)\end{array}$ & $\underset{(\mathrm{mm})}{\mathrm{L}}$ & $\begin{array}{c}\mathrm{P} / \mathrm{L} \\
\left(\mathrm{mmH}_{2} \mathrm{O} / \mathrm{mm}\right)\end{array}$ & $\begin{array}{c}W \\
\left(10^{-4} J\right)\end{array}$ \\
\hline Farinha Padrão & $129,1 b^{*}$ & $38 a$ & $3,45 a$ & $250,7 a$ \\
\hline Farinha com CaSO ${ }_{4} 2 \mathrm{H}_{2} \mathrm{O}$ & $127,9 b$ & $38 a$ & $3,47 a$ & $246,5 a$ \\
\hline Farinha $\operatorname{com} \mathrm{CaCO}_{3}$ & $149,5 a$ & $36 a$ & $4,12 a$ & $291,4 a$ \\
\hline Farinha com CaHPO 4 & $133,6 \mathrm{~b}$ & $37 a$ & $3,60 \mathrm{a}$ & $256,7 \mathrm{a}$ \\
\hline
\end{tabular}

TABELA 6. Extensigrafia das farinhas de trigo Padrão e das enriquecidas de diferentes sais de cálcio.

\begin{tabular}{|c|c|c|c|c|}
\hline AMOSTRAS & $\begin{array}{c}\text { RESISTÉNCIA } \\
\text { MÁXIMA Á } \\
\text { EXTENSÃO } \\
\text { Rm } \\
\text { (UF) } \\
\end{array}$ & $\begin{array}{l}\text { EXTENSIBILIDADE } \\
\text { E } \\
(\mathrm{mm})\end{array}$ & $\begin{array}{c}\text { NÚMERO } \\
\text { PROP ORCIONAL } \\
\text { Rm/E } \\
(\mathrm{UF} / \mathrm{mm}) \\
\end{array}$ & $\begin{array}{l}\text { ÁREA } \\
\left(\mathrm{cm}^{2}\right)\end{array}$ \\
\hline Farinha padrão & $849 a^{*}$ & $74 a$ & $11,5 \mathrm{a}$ & $72,5 a$ \\
\hline Farinha com CaSO ${ }_{4} 2 \mathrm{H}_{2} \mathrm{O}$ & $812 a$ & $68 a$ & $11,9 a$ & $61,8 \mathrm{a}$ \\
\hline Farinha com $\mathrm{CaCO}_{3}$ & $842 a$ & $82 a$ & $10,3 a$ & $79,1 \mathrm{a}$ \\
\hline Farinha com CaHPO & $899 a$ & $77 a$ & $11,8 \mathrm{a}$ & $75,2 \mathrm{a}$ \\
\hline
\end{tabular}

* Médias das colunas seguidas de letras iguais não são significativamente diferentes $(\mathrm{p} \leq 5 \%)$, pelo teste de Tukey.

Os resultados de volume específico e conceito global dos pães franceses elaborados com a farinha e as enriquecidas de sais de cálcio, são apresentados na Tabela 7.

TABELA 7. Volume específico e conceito global do pão francês elaborados com farinha Padrão e farinha enriquecida com diferentes sais de cálcio.

\begin{tabular}{lll}
\hline \multicolumn{1}{c}{ AMOSTRAS } & $\begin{array}{c}\text { VOLUME } \\
\text { ESPECÍFICO } \\
\left(\mathrm{cm}^{3} / \mathrm{g}\right)\end{array}$ & $\begin{array}{c}\text { CONCEITO } \\
\text { GLOBAL }\end{array}$ \\
\hline Pão elaborado com farinha Padrão & $7,13 \mathrm{a}^{*}$ & Muito bom \\
Pão elaborado com farinha com CaSO ${ }_{4} \mathbf{2 H}_{2} \mathrm{O}$ & $7,23 \mathrm{a}$ & Muito bom \\
Pão elaborado com farinha com $\mathrm{CaCO}_{3}$ & $6,73 \mathrm{a}$ & Muito bom \\
Pão elaborado com farinha com $\mathrm{CaHPO}_{4}$ & $7,50 \mathrm{a}$ & Muito bom \\
\hline
\end{tabular}

*médias das colunas seguidas de letra igual não são significativamente diferentes $(\mathrm{p} \leq 5 \%)$, pelo teste de Tukey.

Embora o volume específico não seja significativamente diferente entre as diversas amostras, a farinha enriquecida de $\mathrm{CaHPO}_{4}$ apresentou o maior valor, seguido daquela adicionada de $\mathrm{CaSO}_{4} 2 \mathrm{H}_{2} \mathrm{O}$. Quanto ao conceito global, também não foram observadas diferenças significativas, ou seja, essencialmente não se observa qualquer alteração na granulosidade, textura, simetria e cor da crosta e do miolo, nos pães elaborados com os diferentes sais de cálcio. 


\section{4 - CONCLUSÕES}

Nas condições experimentais realizadas podemos concluir que, o enriquecimento de pão, com niveis de $100 \%$ da IDR de cálcio de adulto, pode ser feito tanto com sulfato, carbonato ou fosfato de cálcio, pois neste nivel de adição tanto os resultados obtidos na reologia da massa quanto nas características do produto final, foram plenamente satisfatórios.

\section{5 - REFERÊNCIAS}

[1] AACC - American Association of Cereal Chemists. Approved methods of AACC, 9a edição, v. I e II, St. Paul, 1995.

[2] ABIP, O pão francês alimenta e pode ser usado em dietas. Disponivel em:< http://www.abip.org.br/padaria/ pt.htm>. Acesso em 12/Out/1999.

[3] BRASIL, Portaria SVS / MS N 33 Ingestão Diária Recomendada (IDR) de vitaminas, minerais e proteínas. Diário Oficial da República Federativa do Brasil, Brasília, DF, 13 de janeiro de 1998.

[4] BAURUFFALDI, R. \& OLIVEIRA, M. N. Fundamentos de tecnologia de alimentos. São Paulo, Atheneu, v. 3, 1998. 567p.

[5] CIACCO, C.F. \& CHANG, Y.K. Como fazer massas. São Paulo, Editora Icone, 1986.

[6] CODEX ALIMENTARIUS. Requisitos Generales. FAO/OMS. Roma. v.1, p. 48-110, 1992.

[7] COKIRER, M. \& LACHANCE, P.A. Added micronutrients, their stability in wheat flour during storage and baking process. The Bakers Digest, v. 49, n. 1, p. 53-57, Feb., 1975.

[8] EL-DASH,A A; CAMARGO, C. O. \& DIAZ, N. M. Fundamentos da tecnologia de panificação. Secretaria da Indústria, Comércio e Tecnologia do Estado de São Paulo, p. 1 $-243,1982$.
[9] EL-DASH,A. A; MAZZARI, M. R. \& GERMANI, R. Tecnologia de farinhas mistas. Uso de farinha mista de trigo e milho na produção de pães. Brasília. EMBRAPA-CTAA. V.1, p. 42-88, 1994.

[10] GERMANI, R.; BENASSI, V. T.; CARVALHO, J. L.; WATANABE, E.; CAMPOS, J. E. \& CARVALHO, C. W. P. Curso de controle de qualidade tecnológica do grão e da farinha de trigo. (apostila). Rio de Janeiro: EMBRAPACTAA, 1997. 60p.

[11] GERMANI, R. \& BENASSI, V. T. Misturas de farinhas de trigo: efeito na elaboração de pão francês e de forma. XVI Congresso Brasileiro de Ciência e Tecnologia de Alimentos v.3, p. 1887-1889, 1998.

[12] IBGE, Instituto Brasileiro de Geografia e Estatística. Pesquisa de orçamentos familiares 1995-1996: Consumo alimentar domiciliar percapita . Rio de Janeiro, V.2, p.21-32. 1998.

[13] NAS / NRC . Proposed Fortification Policy for Cereal-Grain Products. Natinal Academy of Sciences, National Research Council, Washington. DC, 1974, 36p.

[14] NESHEIN, R. D. \& LOKHART, H. B. Cereal Nutrition. In FAST, R. B. \& CALDWELL, E. F. Breakfast cereals and how they are made. AACC, $3^{\text {a }}$ ed, St. Paul, 1993, cap. 11, p. 311- 317.

[15] RANHOTRA, G., LEE, C. \& GELROTH, J. Expanded cereal fortification: bioavailability and functionality (breadmaking) of variious calcium sources. Nutricion Report. v. 22, n. 4, p. 469-475, 1980.

[16] RANHOTRA, G. S.; GELROTH, J. A.; LEINEN, S. D. \& SCHNELLER, F. E. Bioavailability of calcium in breads fortified with different calcium sources. Cereal Chemists. v.74, n . 4, p. 361 - 363, 1997.

[17] RANUM, P. M. Note on levels of nutrients to add under expanded wheat flour fortification/enrichment programs. Cereal Chemistry. v. 57, n. 1, p. $70-72,1980$.

[18] VANNUCCHI, H.; MENEZES, E. W.; CAMPANARA, A O \& LAJOLO, F.M. Cadernos de Nutrição. Sociedade Brasileira de Alimentação e Nutrição. v. 2, p. 101- 102, 1990. 